\title{
Grey Target Decision Method for a Variable Target Centre Based on the Decision Maker's Preferences
}

\author{
Jinshan $\mathrm{Ma}^{1,2}$ \\ ${ }^{1}$ School of Mines, China University of Mining and Technology, Xuzhou, Jiangsu 221116, China \\ ${ }^{2}$ School of Energy Science and Engineering, Henan Polytechnic University, Jiaozuo, Henan 454000, China \\ Correspondence should be addressed to Jinshan Ma; mjscumttf@163.com
}

Received 15 January 2014; Revised 3 April 2014; Accepted 4 April 2014; Published 17 April 2014

Academic Editor: Zhihua Zhang

Copyright (C) 2014 Jinshan Ma. This is an open access article distributed under the Creative Commons Attribution License, which permits unrestricted use, distribution, and reproduction in any medium, provided the original work is properly cited.

In multiattribute grey target decision making, the decision maker (DM) may have certain preferences for some attributes. The impacts of two types of DM's preferences for some attribute values on alternatives were studied. To deal with the attribute preferences of a DM, a generalised grey target decision method was presented. The proposed method required that the index values of all alternatives were not normalised. The target centre index values can be obtained by substituting DM's preference values for some of the original target centre index values as determined by the alternatives themselves. Following this, the proposed generalised method was used to calculate the target centre distances. A case study showed that this method of handling DM's preferences for some attributes was effective.

\section{Introduction}

In multiattribute decision making, the relative optimality of one parameter can be obtained using a grey target decision method by comparison with feasible alternatives without recourse to other standard modes. The grey target decision method has been widely used in many fields since it was proposed by Deng [1]. Over the past few years, many scholars have made progress in this area. Chen and Xie tested the incontinency problem of Deng's grey transformation by simulation [2]. Dang et al. improved the calculation operators of the grey target decision method [3,4]. Some scholars also studied its weight determination [5-7]. The grey target decision method for mixed attributes has also been studied [8-14]. Furthermore, some other theories and methods were introduced to the grey target decision method [1316] which enrich its potential. However, the consideration of the DM's preferences was seldom studied apart from a limited contribution by Zhu and Hipel $[6,16]$. This work expanded the target centre as determined by the alternatives themselves to some indices of the target centre replaced by the DM's preferences. There are two types of preferences: some attribute values were expected to reach their desired levels; however, some other attribute values were regarded as excellent only if they reached some specified values without acquiring the optimal solution. This work assessed the effects of a variable target centre determined partially by the DM's preferences over the available alternatives and presented a new generalised grey target decision method to deal with this problem.

The remainder of this paper is organised as follows: Section 2 introduces the concepts, Section 3 discusses the proposed method, Section 4 presents a case study, and Section 5 is the conclusion.

\section{Preliminaries}

Definition 1. Let $S=\left\{S_{1}, S_{2}, \ldots, S_{n}\right\}$ be an alternative set, let $A=\left\{A_{1}, A_{2}, \ldots, A_{m}\right\}$ be an attribute set, and let $S_{i j}(i=$ $1,2, \ldots, n, j=1,2, \ldots, m)$ be the measure of alternative $S_{i}$ under attribute $A_{j}$, and $J^{+}$and $J^{-}$are benefit type attribute, and cost type attribute, sets, respectively: these form the basic elements of multiattribute decision making.

Remark 2. Based on the theory of grey target decision making, however the method differed from the classical version (the generalised grey target method). Compared to 
the traditional model, the generalised grey target method had two differences: no need to normalise the index values $S_{i j}(i=1,2, \ldots, n, j=1,2, \ldots, m)$ and the difference in the target centre distance calculation.

Definition 3. Let $C^{a}=\left(C_{1}^{a}, C_{2}^{a}, \ldots, C_{m}^{a}\right)$ be the target centre determined by the alternative measure $S_{i j}(i=1,2, \ldots, n, j=$ $1,2, \ldots, m)$, where $C_{j}^{a}$ satisfies

$$
C_{j}^{a}=\left\{\begin{array}{l}
\max \left\{S_{i j}\right\}, S_{i j} \in J^{+} \\
\min \left\{S_{i j}\right\}, S_{i j} \in J^{-}
\end{array} \quad i=1,2, \ldots, n, j=1,2, \ldots, m .\right.
$$

Definition 4. Let $C_{k}^{\text {de }}(k \in\{1,2, \ldots, m\})$ be the DM's desirable preference value, such that the DM's preference value is better than or equal to the optimal index value of alternatives under attribute $A_{j}$, which satisfies

$$
\begin{array}{r}
C_{k}^{\mathrm{de}} \geq \max \left\{S_{i j}\right\}, \\
S_{i j} \in J^{+}, i=1,2, \ldots, n, j=1,2, \ldots, m,
\end{array}
$$

or

$$
\begin{array}{r}
C_{k}^{\mathrm{de}} \leq \min \left\{S_{i j}\right\}, \\
S_{i j} \in J^{-}, i=1,2, \ldots, n, j=1,2, \ldots, m .
\end{array}
$$

Definition 5. Let $C_{k}^{\mathrm{ds}}(k \in\{1,2, \ldots, m\})$ be the DM's selection preference value, such that some index value is regarded as excellent only when it is better than or equal to the value $C_{k}^{\mathrm{ds}}$ given by the DM under attribute $A_{j}$, which satisfies

$$
\begin{aligned}
& \min \left\{S_{i j}\right\}<C_{k}^{\mathrm{ds}}<\max \left\{S_{i j}\right\}, \\
& i=1,2, \ldots, n, j=1,2, \ldots, m .
\end{aligned}
$$

Definition 6. Suppose that the target centre $C^{a}=$ $\left(C_{1}^{a}, C_{2}^{a}, \ldots, C_{m}^{a}\right)$ is decided by $S_{i j}(i=1,2, \ldots, n, j=$ $1,2, \ldots, m)$ and DM's preference value under attribute $A_{k}(k \in\{1,2, \ldots, m\})$ is $C_{k}^{d}(k \in\{1,2, \ldots, m\})$. Then the target centre, determined partially by preference values, becomes $C^{0}=\left(C_{1}^{0}, C_{2}^{0}, \ldots, C_{m}^{0}\right)$, the elements of which are as follows:

$$
C_{j}^{0}= \begin{cases}C_{j}^{a}, & j=1,2, \ldots, m, j \neq k \\ C_{k}^{d}, & k \in\{1,2, \ldots, m\}, j=k\end{cases}
$$

\section{Grey Target Decision Making Method for Variable Target Centre}

3.1. The Impacts of Variable Target Centre on Alternatives. Desirable preferences and selection preferences are two types of attribute preferences for the DM. Different DM's attribute preferences may cause different impacts on alternatives with respect to any grey target decision model. Figure 1 shows the impact of desirable attribute preference on the alternatives.

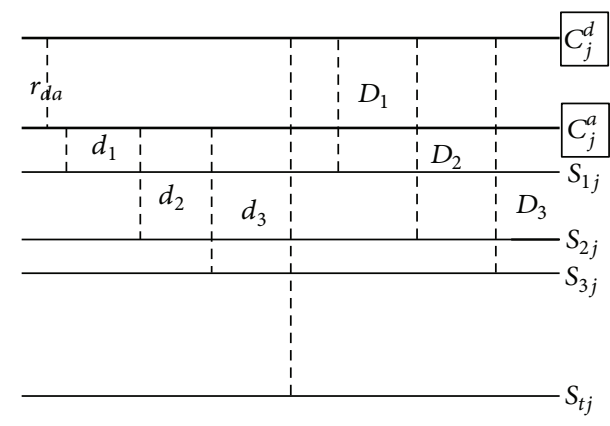

FIgURE 1: The impact of desirable attribute preference on the alternative.

In Figure 1, suppose that $S_{i j}$ which belongs to the benefit type attribute set is the index value under attribute $A_{j}$, and $S_{t j}$ is the worst value. Let $C_{j}^{a}$ and $C_{j}^{d}$ be the target centre indices under attribute $A_{j}$, as determined by feasible alternatives and DM's preferences, respectively. Suppose that $d_{1}, d_{2}$, and $d_{3}$ are the distances of index values $S_{1 j}, S_{2 j}$, and $S_{3 j}$ to $C_{j}^{a}$, respectively, and $D_{1}, D_{2}$, and $D_{3}$ are the distances of index values $S_{1 j}, S_{2 j}$, and $S_{3 j}$ to $C_{j}^{d}$, respectively, while $r_{d a}$ is the difference between $C_{j}^{d}$ and $C_{j}^{a}$. Obviously, the target centre index $C_{j}^{d}$, determined by the desirable attribute preference value, expanded the distances from $d_{1}, d_{2}$, and $d_{3}$ to $D_{1}, D_{2}$, and $D_{3}$, respectively. Figure 2 shows the impact of selection attribute preference on alternatives (the meaning of the parameters in Figure 2 matches that in Figure 1). The target centre index value determined by DM's selection preference is inferior to that of the alternatives, which changes the distances of $S_{1 j}, S_{2 j}$, and $S_{3 j}$ to $C_{j}^{a}$ to the distances of $S_{1 j}$, $S_{2 j}$, and $S_{3 j}$ to $C_{j}^{d}$, such that $d_{1}, d_{2}$, and $d_{3}$ changed to $D_{1}$, $D_{2}$, and $D_{3}$, respectively. It can be seen from Figure 2 that $C_{j}^{d}$ was actually inferior to $S_{1 j}$ and $S_{2 j}$, so there was no meaning attributable to either $D_{1}$ or $D_{2}$. From the perspective of a cluster of indices, the distances of $S_{1 j}$ and $S_{2 j}$ to $C_{j}^{d}$ can be regarded as excellent indices with target centre distances of zero. Only $D_{3}$ denoted the real target centre distances, but its value is less than $d_{3}$ and the reduced value is $r_{d a}$ which is the difference between $C_{j}^{a}$ and $C_{j}^{d}$.

The impacts of target centre determined by different preferences over the alternatives are discussed as follows: assume that $S_{i j}(i=1,2, \ldots, n, j=1,2, \ldots, m)$ is the measure of alternative $S_{i}$ under attribute $A_{j}$ and $S_{i_{0} j}$ and $S_{\left(i_{0}+1\right) j}$ are any two index values. Let $d_{i_{0}}$ and $d_{i_{0}+1}$ be the distances of $S_{i_{0} j}$ and $S_{\left(i_{0}+1\right) j}$ to $C_{j}^{a}$, respectively; then set $d_{i_{0}}<d_{i_{0}+1}$ without affecting the conclusions so that under attribute $A_{j}$ the distances of $S_{i_{0} j}$ and $S_{\left(i_{0}+1\right) j}$ to $C_{j}^{d}$ are $D_{i_{0}}$ and $D_{i_{0}+1}$, respectively: the difference between $C_{j}^{d}$ and $C_{j}^{a}$ is $r_{d a}$. For comparison, the target centre distances of all indices under some attribute must be normalised. The linear method is used to normalise these target centre distances using (12). The following equations are the difference between the two 


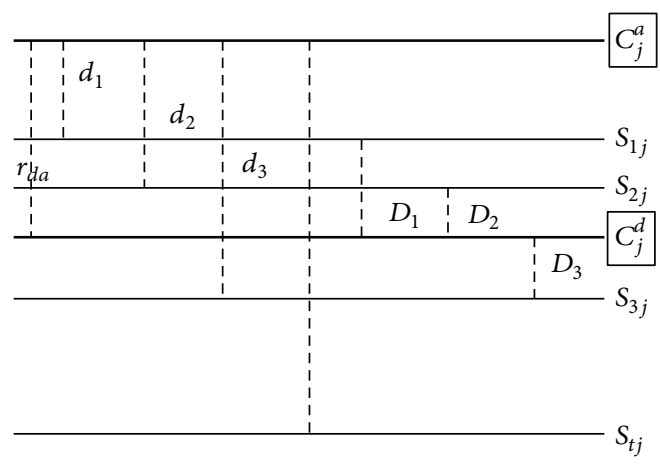

FIGURE 2: The impact of selection attribute preference on the alternative.

alternatives' target centre distances under some attribute for different target centres:

$$
\Delta Z_{a}=\frac{d_{i_{0}+1}}{\sum_{i=1}^{n} d_{i}}-\frac{d_{i_{0}}}{\sum_{i=1}^{n} d_{i}}=\frac{d_{i_{0}+1}-d_{i_{0}}}{\sum_{i=1}^{n} d_{i}}
$$

where $d_{i}$ is the distance of $S_{i j}$ to $C_{j}^{a}$; namely, $d_{i}=\left|C_{j}^{a}-S_{i j}\right|$ :

$$
\Delta Z_{d}=\frac{D_{i_{0}+1}}{\sum_{i=1}^{n} D_{i}}-\frac{D_{i_{0}}}{\sum_{i=1}^{n} D_{i}}=\frac{D_{i_{0}+1}-D_{i_{0}}}{\sum_{i=1}^{n} D_{i}}
$$

where $D_{i}$ is the distance of $S_{i j}$ to $C_{j}^{d}$, which can be calculated by (11).

The target centre $C_{j}^{a}$ determined by alternatives and the target centre $C_{j}^{d}$ determined by DM's preference value have the following relationship:

$$
r_{d a}=C_{j}^{d}-C_{j}^{a}
$$

So (7) can be rewritten as

$$
\begin{aligned}
\Delta Z_{d}= & \frac{r_{d a}+d_{i_{0}+1}}{\sum_{i=1}^{n}\left(r_{d a}+d_{i}\right)} \\
& -\frac{r_{d a}+d_{i_{0}}}{\sum_{i=1}^{n}\left(r_{d a}+d_{i}\right)}=\frac{d_{i_{0}+1}-d_{i_{0}}}{n r_{d a}+\sum_{i=1}^{n} d_{i}} .
\end{aligned}
$$

Compared with (5) and (2), the conclusions may be drawn as follows.

(1) If $r_{d a}>0$, which is the DM's desirable preference, then $\Delta Z_{d}<\Delta Z_{a}$ means the difference of the two alternatives' target centre distances decreased, which implied that the target centre, as determined by desirable preference, can reduce the difference in index values for each alternative.

(2) If $r_{d a}<0$, which is the DM's selection preference, then $\Delta Z_{d}>\Delta Z_{a}$ means the difference of the two alternatives' target centre distances increased, which implied that the target centre, as determined by selection preference, can enlarge the difference in index values for each alternative. However, note that some indices' target centre distances were zero when they were superior to the target centre index implying an indifference to the value of these indices. Therefore, the target centre index, as determined by selection preference, had the potential to act as a "rewarding good and punishing bad" function.

This discussion was based on benefit-type indices; however, the same conclusions may be drawn from consideration of cost-type indices.

3.2. Variable Target Centre Determination. To obtain the target centre combined with the DM's preferences, the target centre, as decided by alternatives, must first be determined. The final target centre was determined by substituting some preference values for the predetermined target centre index values. Note that the predetermined target centre originated from the nonnormalised index matrix. The target centre combined with DM's preferences can be obtained using (1) and (5).

3.3. Target Centre Distance Calculation. In grey target decision making, the optimal alternative is determined by the minimum of all integrated target centre distances. The target centre determined only by the DM's desirable preferences is easy to deal with; however, the target centre combined with the DM's selection preferences may be more complicated. Some index values may be superior to the target centre index values determined by selection preferences, so their index target centre distances were zero, as were all those regarded as excellent values. A new generalised grey target method will be used to solve this problem.

Suppose that the target centre determined by $S_{i j}(i=$ $1,2, \ldots, n, j=1,2, \ldots, m)$ was $C^{a}=\left(C_{1}^{a}, C_{2}^{a}, \ldots, C_{m}^{a}\right)$, so the target centre combined with the DM's preferences can be calculated according to the following steps.

(1) The new index measure $I_{i j}$ can be obtained from $S_{i j}(i=1,2, \ldots, n, j=1,2, \ldots, m)$ compared with the target centre index $C_{j}^{0}(j=1,2, \ldots, m)$ under attribute $A_{j}(j=1,2, \ldots, m)$ :

$I_{i j}= \begin{cases}S_{i j}, & \text { if }\left(S_{i j}<C_{j}^{0}, S_{i j} \in J^{+}\right) \text {or }\left(S_{i j}>C_{j}^{0}, S_{i j} \in J^{-}\right) \\ C_{j}^{0}, & \text { if }\left(S_{i j} \geq C_{j}^{0}, S_{i j} \in J^{+}\right) \text {or }\left(S_{i j} \leq C_{j}^{0}, S_{i j} \in J^{-}\right) .\end{cases}$

(2) Calculate the distance of index value $I_{i j}(i=$ $1,2, \ldots, n, j=1,2, \ldots, m)$ to the target centre index value $C_{j}^{0}(j=1,2, \ldots, m)$ under attribute $A_{j}(j=$ $1,2, \ldots, m)$, using the Hamming distance:

$$
r_{i j}=\left|C_{j}^{0}-I_{i j}\right|, \quad i=1,2, \ldots, n, j=1,2, \ldots, m .
$$

(3) Normalise the index target centre distances of all alternatives for comparability, and the linear normalised method was then used to retain the indices' own characteristics:

$$
z_{i j}=\frac{r_{i j}}{\sum_{i=1}^{n} r_{i j}}, \quad i=1, \ldots, n ; j=1, \ldots, m .
$$


(4) Having obtained the weight $\omega_{j}$ under attribute $A_{j}(j=1,2, \ldots, m)$, the integrated target centre distances for all alternatives can then be calculated using (13):

$$
w_{i}=\omega_{j} z_{i j}, \quad i=1, \ldots, n, j=1, \ldots, m .
$$

3.4. Weight Determination. The attribute weights can be determined by: subjective method, objective method, or comprehensive method. There are many articles contributing to weight determination: the interested reader is referred to the relevant literature $[2-4,10,12]$.

\subsection{Algorithm of Grey Target Decision Making Method Based on the DM's Preferences}

(1) Give the DM's attribute preferences.

(2) Calculate the original target centre for nonnormalised alternatives' matrix of index values.

(3) Achieve the target centre combined with the DM's attribute preferences.

(4) Deal with the index values of all alternatives by the final target centre.

(5) Calculate the distances of all index values to their target centre index values.

(6) Determine the weights of all attributes.

(7) Integrate all of the normalised target centre distances under all attributes for all alternatives, and rank the alternatives according to their integrated target centre distances in ascending order.

\section{Case Study}

4.1. Background and Data. To evaluate ten coal mines' comprehensive safety performances, eight indices including seam $\operatorname{dip}\left({ }^{\circ}\right)$, methane emission rate $\left(\mathrm{m}^{3} / \mathrm{t}\right)$, water inflow $\left(\mathrm{m}^{3} / \mathrm{h}\right)$, spontaneous combustion period (month), ventilating structures qualification rate (\%), equivalent orifice $\left(\mathrm{m}^{2}\right)$, mortality per million tons (person $/ 10^{6} \mathrm{t}$ ), and accident economic loss ( $10^{5}$ Yuan) [17] are denoted by $A_{1}$ to $A_{10}$, and alternatives are denoted by $S_{1}$ to $S_{10}$. The data are shown in Table 1 , the benefit-type attributes are $A_{4}$ to $A_{6}$, and the others are costtype attributes. The DM's attribute preferences are $A_{2}, A_{5}, A_{6}$, and $A_{7}$ with their values set to $0,95,2.0$, and 0.2 , respectively.

\subsection{Decision Making Process}

(1) Calculate the target centre determined by alternatives. The original target centre $C^{a}=(10,3.7,120,12,100,3.6$, $0,300)$ is obtained using (1).

(2) Determine the target centre combined with the DM's preferences.

The final target centre $C^{0}=(10,0,120,12,95,2.0$, $0.2,300)$ combined with the DM's preferences can be determined using (5).
(3) Deal with the index matrix based on target centre $C^{0}$. Use (10) and the original index matrix can be converted to a new index matrix based on target centre $C^{0}$ : the results are shown in Table 2.

(4) Calculate all index target centre distances.

Using (11), all index target centre distances can be calculated as listed in Table 3.

(5) Normalise all index target centre distances.

All index target centre distances can be normalised using (12) with the results shown in Table 4.

(6) Integrate the normalised index target centre distances.

Given $\omega=(0.06,0.15,0.03,0.08,0.12,0.13,0.27,0.14)$, the integrated target centre distances $w=(0.043051,0.110387$, 0.140379, 0.064991, 0.082207, 0.015661, 0.189271, 0.146908, $0.124351,0.186678)$ can be obtained by (13). So the alternatives, in rank order, were $S_{6}>S_{1}>S_{4}>S_{5}>S_{2}>S_{9}>S_{8}>$ $S_{3}>S_{10}>S_{7}$.

Given $\omega=(0.06,0.15,0.03,0.08,0.12,0.13,0.27,0.14)$ without considering the preferences, then $w=(0.055198$, 0.094923, 0.147292, 0.049522, 0.091520, 0.020273, 0.166158, $0.173715,0.119444,0.076746)$ can be obtained by (13). So the alternatives in rank order were $S_{6}>S_{4}>S_{1}>S_{10}>S_{5}>$ $S_{2}>S_{9}>S_{3}>S_{7}>S_{8}$.

4.3. Discussion. The results, considering the attribute preferences of $A_{2}, A_{5}, A_{6}$, and $A_{7}$ with values $0,95,2.0$, and 0.2 , respectively, and the results without considering attribute preferences are shown in Table 5 .

As seen in Table 5, the integrated target centre distances and alternative ranking would change when considering the DM's preferences. With respect to the ranking of the alternatives, most of them changed except for $S_{3}$ and $S_{6}$. Alternative $S_{10}$ changed its ranking from fourth to ninth when not considering preferences and considering preferences: the magnitude of this change indicated that the DM's attributes influenced the decision making with regard to the available alternatives.

\section{Conclusions}

This research proposed a grey target decision method with a variable target centre considering DM's desirable preferences and selection preferences. The study indicated that the target centre determined by desirable preferences could reduce the difference between index values for each alternative, which resulted in indicial clustering. However, the target centre, as determined by selection preference, had the potential to act in a "rewarding good and punishing bad" role. When some index values were superior to the target centre index, these indices were rewarded as excellent values; when some index values were inferior to the target centre index, these indices were punished with a larger difference therefrom. A case study illustrated that the generalised grey target decision method could effectively solve the problem for a target centre determined partially by the DM's preferences. 
TABLE 1: Safety data from coal mines.

\begin{tabular}{|c|c|c|c|c|c|c|c|c|}
\hline$S_{i}$ & $A_{1}$ & $A_{2}$ & $A_{3}$ & $A_{4}$ & $A_{5}$ & $A_{6}$ & $A_{7}$ & $A_{8}$ \\
\hline$S_{1}$ & 21 & 6 & 220 & 12 & 92 & 1.8 & 0.18 & 381 \\
\hline$S_{2}$ & 16 & 3.7 & 200 & 6 & 90 & 1.4 & 0.712 & 564 \\
\hline$S_{3}$ & 26 & 9.2 & 180 & 10 & 88 & 2.7 & 1.34 & 1051.6 \\
\hline$S_{4}$ & 10 & 4 & 260 & 8 & 94 & 1.2 & 0 & 442.5 \\
\hline$S_{5}$ & 30 & 8.2 & 350 & 10 & 96 & 3.6 & 0.641 & 788 \\
\hline$S_{6}$ & 19 & 5 & 130 & 12 & 100 & 2.4 & 0 & 300 \\
\hline$S_{7}$ & 17 & 9.6 & 400 & 6 & 86 & 1.3 & 1.23 & 964.7 \\
\hline$S_{8}$ & 40 & 14 & 600 & 6 & 95 & 2.1 & 1.12 & 885.6 \\
\hline$S_{9}$ & 12 & 12.8 & 120 & 10 & 91 & 1.5 & 0.872 & 839.3 \\
\hline$S_{10}$ & 14 & 5.8 & 155 & 12 & 89 & 1.7 & 0.426 & 617.2 \\
\hline
\end{tabular}

TABLE 2: Index values processed based on final target centre.

\begin{tabular}{|c|c|c|c|c|c|c|c|c|}
\hline$S_{i}$ & $A_{1}$ & $A_{2}$ & $A_{3}$ & $A_{4}$ & $A_{5}$ & $A_{6}$ & $A_{7}$ & $A_{8}$ \\
\hline$S_{1}$ & 21 & 6 & 220 & 12 & 92 & 1.8 & 0.2 & 381 \\
\hline$S_{2}$ & 16 & 3.7 & 200 & 6 & 90 & 1.4 & 0.712 & 564 \\
\hline$S_{3}$ & 26 & 9.2 & 180 & 10 & 88 & 2.0 & 1.34 & 1051.6 \\
\hline$S_{4}$ & 10 & 4 & 260 & 8 & 94 & 1.2 & 0.2 & 442.5 \\
\hline$S_{5}$ & 30 & 8.2 & 350 & 10 & 95 & 2.0 & 0.641 & 788 \\
\hline$S_{6}$ & 19 & 5 & 130 & 12 & 95 & 2.0 & 0.2 & 300 \\
\hline$S_{7}$ & 17 & 9.6 & 400 & 6 & 86 & 1.3 & 1.23 & 964.7 \\
\hline$S_{8}$ & 40 & 14 & 600 & 6 & 95 & 2.0 & 1.12 & 885.6 \\
\hline$S_{9}$ & 12 & 12.8 & 120 & 10 & 91 & 1.5 & 0.872 & 839.3 \\
\hline$S_{10}$ & 14 & 5.8 & 155 & 12 & 89 & 1.7 & 0.426 & 617.2 \\
\hline
\end{tabular}

TABLE 3: All index target centre distances.

\begin{tabular}{|c|c|c|c|c|c|c|c|c|}
\hline$r_{i j}$ & $A_{1}$ & $A_{2}$ & $A_{3}$ & $A_{4}$ & $A_{5}$ & $A_{6}$ & $A_{7}$ & $A_{8}$ \\
\hline$r_{1 j}$ & 11 & 6 & 100 & 0 & 3 & 0.2 & 0 & 81 \\
\hline$r_{2 j}$ & 6 & 3.7 & 80 & 6 & 5 & 0.6 & 0.512 & 264 \\
\hline$r_{3 j}$ & 16 & 9.2 & 60 & 2 & 7 & 0 & 1.14 & 751.6 \\
\hline$r_{4 j}$ & 0 & 4 & 140 & 4 & 1 & 0.8 & 0 & 142.5 \\
\hline$r_{5 j}$ & 20 & 8.2 & 230 & 2 & 0 & 0 & 0.441 & 488 \\
\hline$r_{6 j}$ & 9 & 5 & 10 & 0 & 0 & 0 & 0 & 0 \\
\hline$r_{7 j}$ & 7 & 9.6 & 280 & 6 & 9 & 0.7 & 1.03 & 664.7 \\
\hline$r_{8 j}$ & 30 & 14 & 480 & 6 & 0 & 0 & 0.92 & 585.6 \\
\hline$r_{9 j}$ & 2 & 12.8 & 0 & 2 & 4 & 0.5 & 0.672 & 539.3 \\
\hline$r_{10 j}$ & 4 & 5.8 & 35 & 0 & 6 & 0.3 & 0.226 & 317.2 \\
\hline
\end{tabular}

TABLE 4: Normalised index target centre distances.

\begin{tabular}{|c|c|c|c|c|c|c|c|c|}
\hline$Z_{i j}$ & $A_{1}$ & $A_{2}$ & $A_{3}$ & $A_{4}$ & $A_{5}$ & $A_{6}$ & $A_{7}$ & $A_{8}$ \\
\hline$Z_{1 j}$ & 0.104762 & 0.076628 & 0.070671 & 0 & 0.085714 & 0.064516 & 0 & 0.021127 \\
\hline$Z_{2 j}$ & 0.057143 & 0.047254 & 0.056537 & 0.214286 & 0.142857 & 0.193548 & 0.103623 & 0.068859 \\
\hline$Z_{3 j}$ & 0.152381 & 0.117497 & 0.042403 & 0.071429 & 0.2 & 0 & 0.230723 & 0.196041 \\
\hline$Z_{4 j}$ & 0 & 0.051086 & 0.09894 & 0.142857 & 0.028571 & 0.258065 & 0 & 0.037168 \\
\hline$Z_{5 j}$ & 0.190476 & 0.104725 & 0.162544 & 0.071429 & 0 & 0 & 0.089253 & 0.127286 \\
\hline$Z_{6 j}$ & 0.085714 & 0.063857 & 0.007076 & 0 & 0 & 0 & 0 & 0 \\
\hline$Z_{7 j}$ & 0.066667 & 0.122605 & 0.19788 & 0.214286 & 0.257143 & 0.225806 & 0.20846 & 0.173374 \\
\hline$Z_{8 j}$ & 0.285714 & 0.178799 & 0.339233 & 0.214286 & 0 & 0 & 0.186197 & 0.152743 \\
\hline$Z_{9 j}$ & 0.019048 & 0.163474 & 0 & 0.071429 & 0.114286 & 0.16129 & 0.136005 & 0.140666 \\
\hline$Z_{10 j}$ & 0.038095 & 0.074074 & 0.024735 & 0 & 0.171429 & 0.96774 & 0.04574 & 0.082736 \\
\hline
\end{tabular}


TABLE 5: Alternatives ranked either with, or without, consideration of preferences.

\begin{tabular}{lccccc}
\hline$S_{i}$ & $Z_{i j}$ (no preferences) & Ranking (no preferences) & $Z_{i j}$ (preferences) & Ranking (preferences) & Ranking changes \\
\hline$S_{1}$ & 0.055198 & 3 & 0.043051 & 2 & -1 \\
$S_{2}$ & 0.094923 & 6 & 0.110387 & 5 & -1 \\
$S_{3}$ & 0.147292 & 8 & 0.140379 & 3 & 0 \\
$S_{4}$ & 0.049522 & 2 & 0.064991 & 4 & +1 \\
$S_{5}$ & 0.091520 & 5 & 0.082207 & 1 & -1 \\
$S_{6}$ & 0.020273 & 1 & 0.015661 & 7 & 0 \\
$S_{7}$ & 0.166158 & 9 & 0.189271 & 6 & -1 \\
$S_{8}$ & 0.173715 & 10 & 0.124351 & 9 & -1 \\
$S_{9}$ & 0.119444 & 7 & 0.186678 & 9 & +5 \\
$S_{10}$ & 0.076746 & 4 & & & +1 \\
\hline
\end{tabular}

\section{Conflict of Interests}

The author declares that there is no conflict of interests regarding the publication of this paper.

\section{Acknowledgments}

The author thanks the Key Research Project of Science and Technology of Henan Province for its support (Grant no. 13B620033), the Natural Science Foundation of the Education Department of Henan Province (Grant no. 2011B620001), and Henan Coal Mine Safety Production Technology Development Project (Grant no. H09-50). The author is also grateful to the editors and the anonymous reviewers for their comments and suggestions for improving the quality of this paper.

\section{References}

[1] J. L. Deng, Grey System Theory, Huazhong University of Science and Technology Press, Wuhan, China, 2002.

[2] Y.-M. Chen and H. Xie, "Test of the inconsistency problem on Deng's grey transformation by simulation," Systems Engineering and Electronics, vol. 29, no. 8, pp. 1285-1287, 2007.

[3] Y. G. Dang, G. F. Liu, J. P. Wang, and B. Liu, "Multi-attribute decision model of grey target considering weights," Statistics and Decision, no. 3, pp. 29-30, 2004.

[4] J. Song, Y.-G. Dang, and Z.-X. Wang, "Multi-attribute decision model of grey target based on majorant operator of "rewarding good and punishing bad'"' Systems Engineering and Electronics, vol. 32, no. 6, pp. 1229-1232, 2010.

[5] Z.-X. Wang, Y.-G. Dang, and H. Yang, "Improvements on decision method of grey target," Systems Engineering and Electronics, vol. 31, no. 11, pp. 2634-2636, 2009.

[6] J. Zhu and K. W. Hipel, "Multiple stages grey target decision making method with incomplete weight based on multigranularity linguistic label," Information Sciences, no. 212, pp. 15-32, 2012.

[7] S.-F. Liu, W.-F. Yuan, and K.-Q. Sheng, "Multi-attribute intelligent grey target decision model," Control and Decision, vol. 25, no. 8, pp. 1159-1163, 2010.

[8] Y. G. Dang, S. F. Liu, and B. Liu, "Study on the multi-attribute decision model of grey target based on interval number," Engineering Science, vol. 7, no. 8, pp. 31-35, 2005.
[9] C. G. Shen, Y. G. Dang, and L. L. Pei, "Hybrid multi-attribute decision model of grey target," Statistics and Decision, no. 12, pp. 17-20, 2010.

[10] D. Luo, "Multi-objective grey target decision model based on positive and negative clouts," Control and Decision, vol. 28, no. 2, pp. 241-246, 2013.

[11] D. Luo and X. Wang, "The multi-attribute grey target decision method for attribute value within three-parameter interval grey number," Applied Mathematical Modelling, vol. 36, no. 5, pp. 1957-1963, 2012.

[12] J. Song, Y.-G. Dang, Z.-X. Wang, and K. Zhang, "New decision model of grey target with both the positive clout and the negative clout," System Engineering-Theory \& Practice, vol. 30, no. 10, pp. 1822-1827, 2010.

[13] Y. Liu, F. Jeffrey, S. F. Liu, and J. S. Liu, "Multi-objective grey target decision-making based on prospect theory," Control and Decision, vol. 28, no. 3, pp. 345-350, 2013.

[14] B. Zeng, S. F. Liu, C. Li, and J. M. Chen, "Grey target decisionmaking model of interval grey number based on cobweb area," Systems Engineering and Electronics, vol. 35, no. 11, pp. 23292334, 2013.

[15] J. J. Zhu, L. L. Zhang, Y. H. Liang, and P. Li, “Grey target decision method based on uncertain evidence aggregation under conflict interest participants," Control and Decision, vol. 27, no. 7, pp. 1037-1046, 2012.

[16] H. H. Wang, J. Zhu, and F. Z. Geng, "Grey target cluster decision method on linguistic evaluation case-based," Systems Engineering-Theory \& Practice, vol. 33, no. 12, pp. 3172-3181, 2013.

[17] S. G. Cao, A. M. Xu, Y. B. Liu, and L. Q. Zhang, "Comprehensive assessment of security in coal mines based on grey relevance analysis," Journal of Mining \& Safety Engineering, vol. 24, no. 2, pp. 141-145, 2007. 


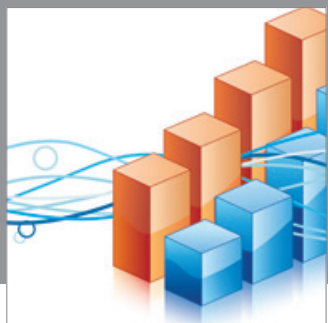

Advances in

Operations Research

mansans

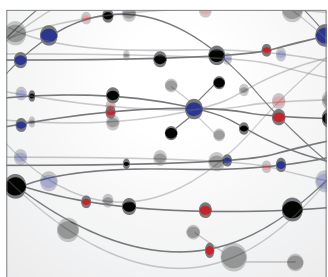

The Scientific World Journal
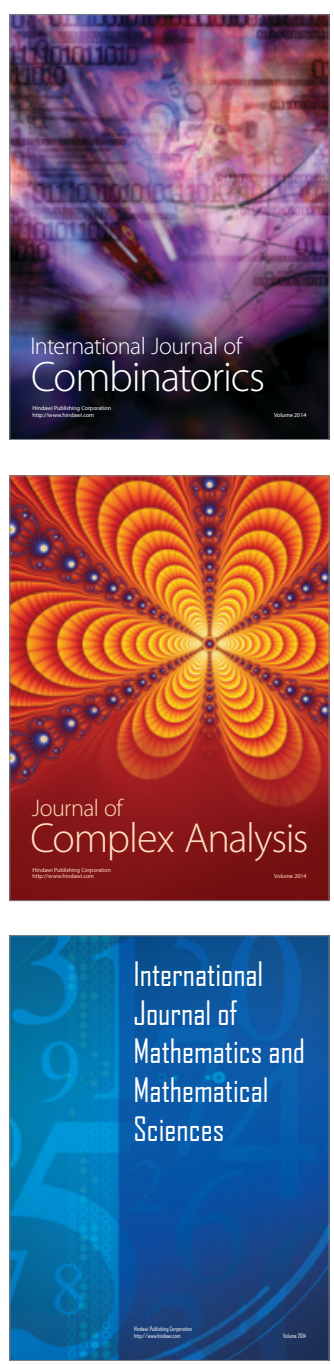
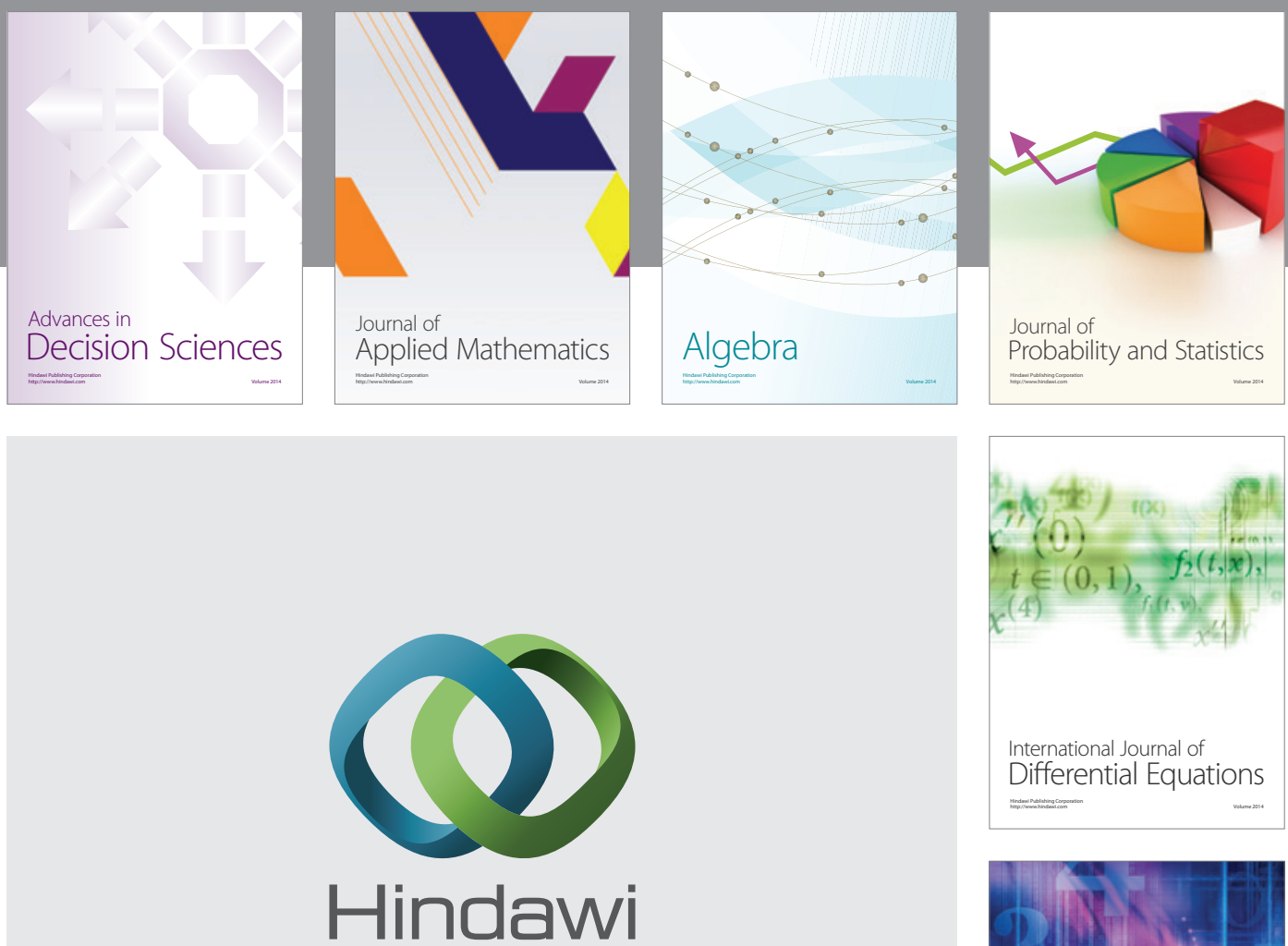

Submit your manuscripts at http://www.hindawi.com
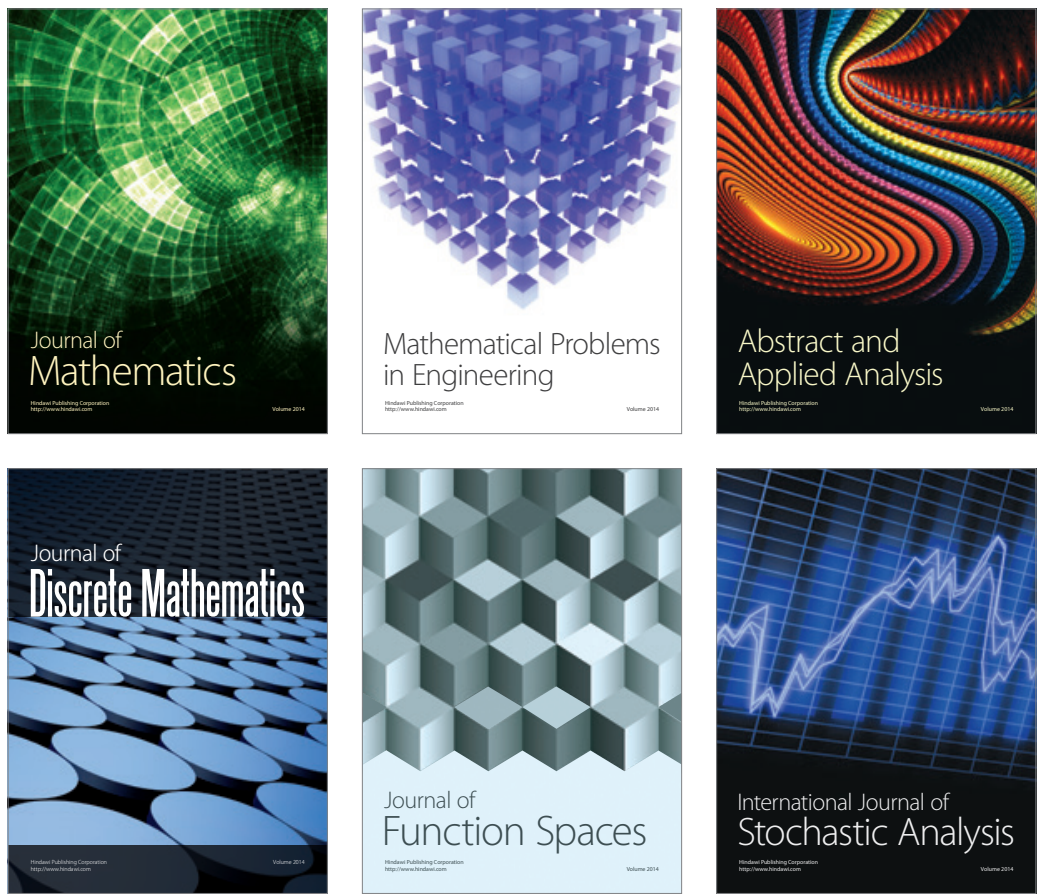

Journal of

Function Spaces

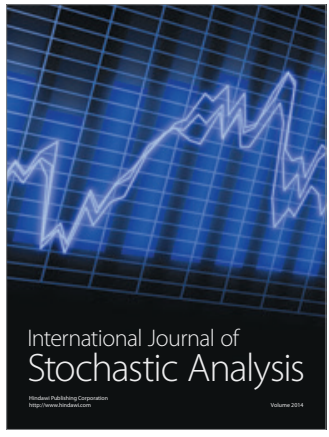

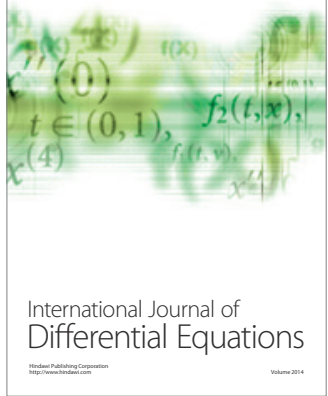
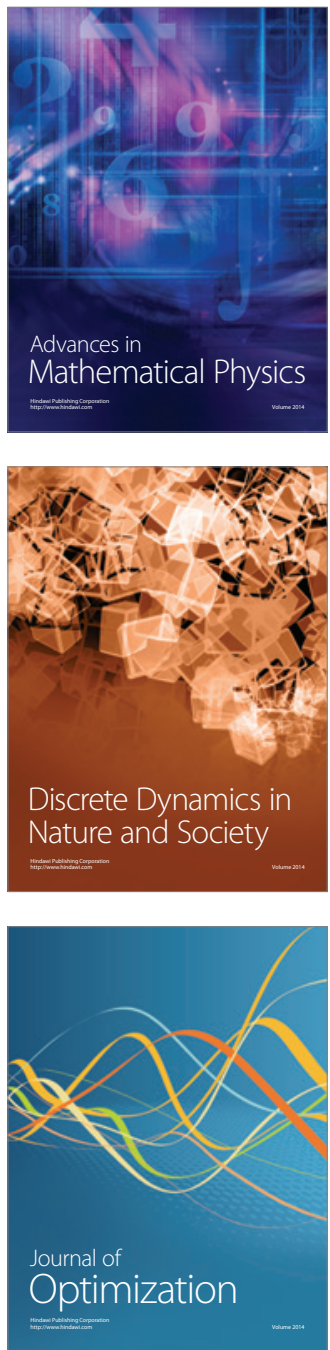